\title{
ON A NONLINEAR ABSTRACT VOLTERRA EQUATION
}

\author{
ETIENNE EMMRICH AND GUY VALLET
}

Communicated by Neville Ford

\begin{abstract}
Existence of solutions is shown for equations of the type $A v+B(K G v, v)=f$, where $A, B$ and $G$ are possibly nonlinear operators acting on a Banach space $V$, and $K$ is a Volterra operator of convolution type. The proof relies on the convergence of a suitable time discretization scheme.
\end{abstract}

1. Introduction. We are concerned with nonlinear Volterra equations of the type

$$
A v+B(K G v, v)=f \quad \text { in }(0, T),
$$

with

$$
(K G v)(t)=u_{0}+\int_{0}^{t} k(t-s) G v(s) d s,
$$

where $(0, T)$ is the time interval under consideration and where $u_{0}$ and $f$ are given data of the problem. Let $V$ be a real, reflexive, separable Banach space with its dual $V^{\prime}$. The operator $A: V \rightarrow V^{\prime}$ is assumed to be hemicontinuous, monotone and $p$-coercive, the operator $B: V \times V \rightarrow V^{\prime}$ is strongly continuous and satisfies a certain growth condition, and the operator $G: V \rightarrow V$ is weakly continuous with linear growth.

2010 AMS Mathematics subject classification. Primary 45D05, 47J35, 65J08.

Keywords and phrases. Nonlinear Volterra equation, time discretization, existence.

The authors acknowledge funding by CRC 910 under project A8, DFG.

Received by the editors on March 14, 2015, and in revised form on September 5, 2015. 
Let us briefly discuss the type of integral kernels $k$ we have in mind. The simplest case is $k \equiv 1$. Problem (1.1) can then be written as

$$
A G^{-1}\left(u-u_{0}\right)^{\prime}+B\left(u, G^{-1}\left(u-u_{0}\right)^{\prime}\right)=f \quad \text { in }(0, T),
$$

if $G$ is invertible (the prime' denotes the time derivative). Problems of this type have been studied in Bauzet and Vallet [1] as well as in Emmrich and Vallet [6]. Such problems of Barenblatt type arise in the description of nonlinear and anomalous diffusion. A typical kernel that arises in many applications is a kernel of exponential type $k(z) \sim \mathrm{e}^{-\lambda z}$ $(\lambda>0)$. Problem (1.1) then reads as

$$
A v+B(u, v)=f \quad \text { in }(0, T)
$$

with

$$
\left(u-u_{0}\right)^{\prime}+\lambda\left(u-u_{0}\right)=G v .
$$

Another typical example is a weakly singular kernel $k(z) \sim z^{-1+\alpha}$ with $\alpha \in(0,1)$. This corresponds to the Riemann-Liouville fractional integral

$$
\left(J^{\alpha} w\right)(t)=\frac{1}{\Gamma(\alpha)} \int_{0}^{t}(t-s)^{-1+\alpha} w(s) d s, \quad t \in[0, T] .
$$

The Riemann-Liouville fractional derivative is then defined by $D^{\beta} u=$ $D^{1} J^{1-\beta} u\left(\beta \in(0,1) ; D^{1}\right.$ denotes the full time derivative), and one easily finds that $D^{\alpha} J^{\alpha} u=u(\alpha \in(0,1))$. For more details and applications, we refer to Diethelm [5], Hilfer [8], Kilbas, et al., [9], Podlubny [12]; see also Lubich $[\mathbf{1 0}, \mathbf{1 1}]$ and the references cited therein for numerical aspects. Problem (1.1) can then also be written as

$$
A v+B(u, v)=f \quad \text { in }(0, T)
$$

with

$$
D^{\alpha}\left(u-u_{0}\right)=G v .
$$

Later we shall need that $k \in L^{p^{\prime}}(0, T)$, where $p$ is the Lebesgue exponent determining the coercivity of $A$ and $p^{\prime}$ denotes the conjugate exponent to $p$.

Abstract nonlinear Volterra equations have been studied by many authors, we refer to the monographs Gripenberg, et al. [7] and 
Prüß [13] and the references cited therein as well as to Corduneanu [2, 3 ]. It is worth mentioning that our results do not require any positivity assumption on the kernel (often, kernels of positive type or completely monotone kernels are considered), and we may also allow kernels of the type $k(z) \sim \mathrm{e}^{-\lambda z} \cos z$ or $k$ being the difference of two weakly singular kernels. Moreover, the problem under consideration here differs from results known on nonlinear Volterra equations insofar as both the operators $A$ and $B$ may be nonlinear and as the operator $B$ describes a nonlinear coupling.

The method for proving existence of a solution is based on a time discretization. Let us consider an equidistant time grid with abscissae $t_{n}=n \tau(n=0,1, \ldots, N \in \mathbb{N})$ and constant step size $\tau=T / N$. We look for approximations $v^{n} \approx v\left(t_{n}\right)$ such that

$$
A v^{n}+B\left(u^{n}, v^{n}\right)=f^{n}, \quad n=1,2, \ldots, N,
$$

with

$$
u^{n}=u^{0}+\tau \sum_{j=1}^{n} \gamma_{n-j+1} G v^{j}, \gamma_{i}=\frac{1}{\tau} \int_{t_{i-1}}^{t_{i}} k(s) d s \quad(i=1,2, \ldots, N),
$$

where $u^{0} \approx u_{0}$ and $\left\{f^{n}\right\}_{n=1}^{N} \approx f$ are given approximations. This discretization is implicit in the first argument of $B$.

The idea behind the numerical scheme above is a simple product quadrature. For $t \in\left(t_{n-1}, t_{n}\right]$, we employ the approximation

$$
\begin{aligned}
\int_{0}^{t} k(t-s) w(s) d s & \approx \int_{0}^{t_{n}} k\left(t_{n}-s\right) w(s) d s=\sum_{j=1}^{n} \int_{t_{j-1}}^{t_{j}} k\left(t_{n}-s\right) w(s) d s \\
& \approx \sum_{j=1}^{n} \int_{t_{j-1}}^{t_{j}} k\left(t_{n}-s\right) d s w\left(t_{j}\right)=: \tau \sum_{j=1}^{n} \gamma_{n-j+1} w\left(t_{j}\right),
\end{aligned}
$$

where

$\gamma_{n-j+1}=\frac{1}{\tau} \int_{t_{j-1}}^{t_{j}} k\left(t_{n}-s\right) d s=\frac{1}{\tau} \int_{t_{n-j}}^{t_{n-j+1}} k(s) d s=\int_{0}^{1} k((n-j+\sigma) \tau) d \sigma$.

Considering a sequence of time discrete problems (1.2) with $\tau \rightarrow 0$, we show convergence of (a subsequence of) the sequence of approximate solutions in a suitable sense. The limit is then identified as a solution to $(1.1)$. 
In the course of the proof of convergence, it turns out that the unique solvability of a corresponding auxiliary problem (see Assumption (AB) below) is crucial. This uniqueness assumption is, of course, a restriction of the class of possible problems we can deal with. Such a restriction, however, is expected since (1.1), interpreted as an operator equation posed on a suitable Banach space of time-dependent abstract functions with values in $V$, misses standard assumptions for proving solvability such as pseudomonotonicity of the governing operator. Let us emphasize that the strong continuity of $B: V \times V \rightarrow V^{\prime}$ does not imply strong continuity of the corresponding Nemytskii operator acting on Bochner integrable functions with values in $V$.

The paper is organized as follows. We close this section by introducing some notation and providing a preliminary result concerning the integral operator. The main result together with all the structural assumptions is then provided in Section 2. The time discrete problem is studied in Section 3. The proof of the main result is finally presented in Section 4.

1.1. Notation and preliminaries. By $L^{r}(0, T ; V)(r \in[1, \infty])$, we denote the usual Bochner-Lebesgue spaces equipped with the standard norm. Here, $(V,\|\cdot\|)$ is a real, reflexive, separable Banach space with its dual $\left(V^{\prime},\|\cdot\|_{V^{\prime}}\right)$ and the duality pairing denoted by $\langle\cdot, \cdot\rangle$. Note that $V^{\prime}$ is reflexive and separable since $V$ is also. Denoting by $r^{\prime}=r /(r-1)$ the conjugate of $r \in(1, \infty)$ with $r^{\prime}=\infty$ if $r=1$, we have $\left(L^{r}(0, T ; V)\right)^{\prime}=L^{r^{\prime}}\left(0, T ; V^{\prime}\right)$ if $r \in[1, \infty)$; the duality pairing is given by

$$
\langle g, v\rangle=\int_{0}^{T}\langle g(t), v(t)\rangle d t .
$$

Moreover, $L^{r}(0, T ; V)$ is reflexive if $r \in(1, \infty)$, and $L^{1}\left(0, T ; V^{\prime}\right)$ is separable, see, e.g., Diestel and Uhl [4] for more details.

The norm in a Banach space $X$ will always be denoted by $\|\cdot\|_{X}$, except for the norm in $V$, where we omit the subscript $V$. We make use of the convention $\sum_{j=1}^{0} z_{j}=0$ for whatsoever $z_{j}$. By $c$, we denote a generic positive constant (that is always independent of the time step size).

Without proof, we give the following result, which is classical for real-valued functions (see, e.g., Gripenberg, et al. [7, Theorem 2.2, 
page 39]) but easily extends to Banach-space-valued functions.

Proposition 1.1. Let $p \in(1, \infty)$ and $u_{0} \in V$. If $k \in L^{1}(0, T)$ then $K$ with

$$
(K w)(t)=u_{0}+\int_{0}^{t} k(t-s) w(s) d s, \quad t \in[0, T],
$$

maps $L^{p}(0, T, V)$ into $L^{p}(0, T, V)$ and is affine-linear and bounded. If $k \in L^{p^{\prime}}(0, T)$, then $K$ maps $L^{p}(0, T, V)$ into $C([0, T], V)$ and is affinelinear and bounded.

2. Main result: Existence via time discretization. Let us start by stating the main assumptions on the operators appearing as well as the integral kernel. Let $p \in(1, \infty)$ be suitably given, and let $(V,\|\cdot\|)$ be a real, reflexive, separable Banach space with dual $\left(V^{\prime},\|\cdot\|_{V^{\prime}}\right)$.

Assumption (A). The operator $A: V \rightarrow V^{\prime}$ is

(i) hemicontinuous, i.e., the mapping $\theta \mapsto\langle A(u+\theta v), w\rangle:[0,1] \rightarrow \mathbb{R}$ is continuous for all $u, v, w \in V$,

(ii) monotone, i.e., $\langle A v-A w, v-w\rangle \geq 0$ for all $v, w \in V$, and

(iii) coercive in the sense that there exist $\mu>0, \lambda \geq 0$ such that, for all $v \in V$,

$$
\langle A v, v\rangle \geq \mu\|v\|^{p}-\lambda
$$

Assumption (B). The operator $B: V \times V \rightarrow V^{\prime}$ is

(i) strongly continuous, i.e., in $V \times V$ weakly convergent sequences are mapped into in $V^{\prime}$ strongly convergent sequences and

(ii) there exist $c>0, q \in[0, p-1)$ such that, for all $u, v \in V$,

$$
\|B(u, v)\|_{V^{\prime}} \leq c\left(1+\|u\|^{p-1}+\|v\|^{q}\right) .
$$

Assumption (AB). For each $u \in V$ and $b \in V^{\prime}$ there exists at most one solution $v \in V$ such that

$$
A v+B(u, v)=b \quad \text { in } V^{\prime} .
$$


Assumption (G). The operator $G: V \rightarrow V$ is

(i) weakly continuous, i.e., in $V$ weakly convergent sequences are mapped into in $V$ weakly convergent sequences and

(ii) there exists $c>0$ such that, for all $v \in V$,

$$
\|G v\| \leq c(1+\|v\|) .
$$

Assumption (k). Let $k \in L^{p^{\prime}}(0, T)$.

Remark 2.1. We shall remark that the operators $A, B$ and $G$ can be extended, as usual, to operators acting on functions defined on $[0, T]$ and taking values in $V$.

Since the operator $A: V \rightarrow V^{\prime}$ is hemicontinuous and monotone, it is also demicontinuous (i.e., it maps strongly into weakly convergent sequences) and thus maps, in view of the separability of $V$ and the theorem of Pettis (see, e.g., Diestel and Uhl [4, Theorem 2, page 42]), a Bochner measurable function $v:[0, T] \rightarrow V$ into a Bochner measurable function $A v:[0, T] \rightarrow V^{\prime}$.

In view of the continuity of the operator $B: V \times V \rightarrow V^{\prime}$ and (2.1), it maps $L^{p}(0, T ; V) \times L^{p}(0, T ; V)$ into $L^{p^{\prime}}\left(0, T ; V^{\prime}\right)$.

Since $G: V \rightarrow V$ is weakly continuous and $V$ is separable, Petti's theorem again provides that $G$ maps Bochner measurable functions into Bochner measurable functions. In view of the linear growth of $G$, we find that $G$ maps $L^{p}(0, T ; V)$ into itself.

As we have not made an assumption on the growth of the operator $A$, it does not need to map $L^{p}(0, T ; V)$ into its dual. Note that the above assumptions do not imply that the operator $B: L^{p}(0, T ; V) \times$ $L^{p}(0, T ; V) \rightarrow L^{p^{\prime}}\left(0, T ; V^{\prime}\right)$ is strongly continuous. This is one main difficulty in the course of the proof of the main result.

Further note that the existence of a solution to (2.2) easily follows from standard results from the theory of monotone operators (see, e.g., Roubíček [14, Proposition 2.17, page 40]).

Let us now state the main result and start with the definition of a solution. 
Definition 2.2 (Solution). Let $u_{0} \in V$ and $f \in L^{p^{\prime}}\left(0, T ; V^{\prime}\right)$ be given. A function $v \in L^{p}(0, T ; V)$ is said to be a solution to (1.1) if (1.1a) holds true in $L^{p^{\prime}}\left(0, T ; V^{\prime}\right)$.

For the discretization in time, let $\left\{N_{\ell}\right\}$ be a sequence of integers such that $N_{\ell} \rightarrow \infty$ as $\ell \rightarrow \infty$. We then consider the sequence of time discrete problems (1.2) with step size $\tau_{\ell}=T / N_{\ell}$, starting value $u_{\ell}^{0} \in V$, and right-hand side $\left\{f^{n}\right\}_{n=1}^{N_{\ell}} \subset V$ given by

$$
f^{n}:=\frac{1}{\tau_{\ell}} \int_{t_{n-1}}^{t_{n}} f(t) d t .
$$

Often we do not call explicitly the dependence of $f^{n}$ and the time instances $t_{n}$ on $\ell$.

Denoting by $\left\{v^{n}\right\}_{n=1}^{N_{\ell}} \subset V$ the solution to (1.2) with step size $\tau_{\ell}$, let $v_{\ell}$ be the piecewise constant function with $v_{\ell}(t)=v^{n}$ for $t \in\left(t_{n-1}, t_{n}\right]$ $\left(n=1,2, \ldots, N_{\ell}\right)$.

The main result reads as follows.

Theorem 2.3 (Existence via convergence of a time discretization). Let Assumptions (A), (B), (AB), (G) and (k) be satisfied. Let $u_{0} \in V$ and $f \in L^{p^{\prime}}\left(0, T ; V^{\prime}\right)$ be given. Moreover, let $\left\{u_{\ell}^{0}\right\} \subset V$ be such that

$$
u_{\ell}^{0} \longrightarrow u_{0} \quad \text { in } \quad V \text { as } \ell \rightarrow \infty \text {. }
$$

Then there is a subsequence (still denoted by $\ell$ ) such that, as $\ell \rightarrow$ $\infty$, the sequence $\left\{v_{\ell}\right\}$ of piecewise constant interpolations of the time discrete solutions to (1.2) with (2.3) converges weakly in $L^{p}(0, T ; V)$; the limit $v$ is a solution to (1.1) in the sense of Definition 2.2.

If $f \in L^{\infty}\left(0, T ; V^{\prime}\right)$ then $v \in L^{\infty}(0, T ; V)$ and $(1.1 a)$ holds in $L^{\infty}\left(0, T ; V^{\prime}\right)$.

The proof will be prepared by the following section on properties of the time discrete solution.

3. The time discrete problem: Solvability and a priori estimates. In this section, we study the time discretization (1.2) for a given time step $\tau>0$. 
Proposition 3.1 (Time discrete problem). Let Assumptions (A), (B), (AB) and (k) be fulfilled. Let $u^{0} \in V$ and $\left\{f^{n}\right\}_{n=1}^{N} \subset V^{\prime}$ be given. Then, if $\tau$ is sufficiently small, there is a solution $\left\{v^{n}\right\}_{n=1}^{N} \subset V$ to (1.2), and the following a priori estimates hold true for $n=1,2, \ldots, N$ :

$$
\begin{aligned}
&\left\|u^{n}\right\|^{p}+\tau \sum_{j=1}^{n}\left\|v^{j}\right\|^{p} \leq c\left(1+\left\|u^{0}\right\|^{p}+\tau \sum_{j=1}^{n}\left\|f^{j}\right\|_{V^{\prime}}^{p}\right), \\
& \tau \sum_{j=1}^{n}\left\|A v^{j}\right\|_{V^{\prime}}^{p^{\prime}}+\tau \sum_{j=1}^{n}\left\|B\left(u^{j}, v^{j}\right)\right\|_{V^{\prime}}^{p^{\prime}} \\
& \leq c\left(1+\left\|u^{0}\right\|^{p}+\tau \sum_{j=1}^{n}\left\|f^{j}\right\|_{V^{\prime}}^{p}\right) .
\end{aligned}
$$

Proof. Existence of a solution follows step-by-step. Let $\left\{v^{j}\right\}_{j=1}^{n-1} \subset V$ be given if $n \geq 2$ (in the case $n=1$ only $u^{0}$ is given). Then $v^{n} \in V$ $(n=1,2, \ldots, N)$ is the solution of the problem

$$
A v^{n}+B\left(\bar{u}^{n-1}+\tau \gamma_{1} G v^{n}, v^{n}\right)=f^{n},
$$

where

$$
\bar{u}^{n-1}=u^{0}+\tau \sum_{j=1}^{n-1} \gamma_{n-j+1} G v^{j} \in V
$$

is given. The mapping

$$
D_{n}: V \longrightarrow V^{\prime}, \quad D_{n} v=A v+B\left(\bar{u}^{n-1}+\tau \gamma_{1} G v, v\right),
$$

is the sum of a hemicontinuous, monotone and a strongly continuous mapping and is coercive if $\tau$ is sufficiently small. Indeed, we find that

$$
\begin{aligned}
&\left\langle D_{n} v, v\right\rangle \geq \mu\|v\|^{p}-\lambda-\left\|B\left(\bar{u}^{n-1}+\tau \gamma_{1} G v, v\right)\right\|_{V^{\prime}}\|v\| \\
& \geq \mu\|v\|^{p}-\lambda-c\left(1+\left\|\bar{u}^{n-1}+\tau \gamma_{1} G v\right\|^{p-1}+\|v\|^{q}\right)\|v\| \\
& \geq \mu\|v\|^{p}-\lambda-c\left(1+\left\|\bar{u}^{n-1}\right\|^{p-1}+\left|\tau \gamma_{1}\right|^{p-1}\|G v\|^{p-1}+\|v\|^{q}\right)\|v\| \\
& \geq \mu\|v\|^{p}-\lambda-c\left(1+\left\|\bar{u}^{n-1}\right\|^{p-1}+c\left|\tau \gamma_{1}\right|^{p-1}\right. \\
&\left.\left(1+\|v\|^{p-1}\right)+\|v\|^{q}\right)\|v\| .
\end{aligned}
$$


Since $\left|\tau \gamma_{1}\right| \leq \int_{0}^{\tau}|k(s)| d s$, Young's inequality yields

$$
\left\langle D_{n} v, v\right\rangle \geq \frac{\mu}{2}\|v\|^{p}-\lambda-c\left(1+\left\|\bar{u}^{n-1}\right\|^{p}\right)-c\left|\tau \gamma_{1}\right|^{p-1}\|v\|^{p},
$$

which shows coercivity if $\tau$ is sufficiently small such that

$$
c\left(\int_{0}^{\tau}|k(s)| d s\right)^{p-1} \leq \frac{\mu}{4} .
$$

Note that the constant $c$ here is the constant that appears in (3.2) in front of $\left|\tau \gamma_{1}\right|^{p-1}\|v\|^{p}$ and is independent of $\tau$. We are now in the position to apply standard results from the theory of monotone operators (see, e.g., Roubíček [14, Proposition 2.17, page 40]) to get existence of $v^{n} \in V$.

Similarly to the coercivity, we obtain the a priori estimates asserted. Indeed, we test (1.2a) by $v^{n}$ and obtain, with (3.2) and (3.3)

$$
\frac{\mu}{4}\left\|v^{n}\right\|^{p}-\lambda-c\left(1+\left\|\bar{u}^{n-1}\right\|^{p}\right) \leq\left\langle D_{n} v^{n}, v^{n}\right\rangle=\left\langle f^{n}, v^{n}\right\rangle .
$$

This yields, together with Assumption (G) and with $\left|\tau \gamma_{n-j+1}\right| \leq$ $\int_{t_{n-j}}^{t_{n-j+1}}|k(z)| d z$

$$
\begin{aligned}
\frac{\mu}{8}\left\|v^{n}\right\|^{p} & \leq c\left(1+\left\|f^{n}\right\|_{V^{\prime}}^{p^{\prime}}+\left\|\bar{u}^{n-1}\right\|^{p}\right) \\
& \leq c\left(1+\left\|f^{n}\right\|_{V^{\prime}}^{p^{\prime}}+\left\|u^{0}\right\|^{p}+\left(\sum_{j=1}^{n-1}\left|\tau \gamma_{n-j+1}\right|\left(1+\left\|v^{j}\right\|\right)\right)^{p}\right) \\
& \leq c\left(1+\left\|f^{n}\right\|_{V^{\prime}}^{p^{\prime}}+\left\|u^{0}\right\|^{p}+\|k\|_{L^{1}(0, T)}^{p}+\left(\sum_{j=1}^{n-1}\left|\tau \gamma_{n-j+1}\right|\left\|v^{j}\right\|\right)^{p}\right) \\
& \leq c\left(1+\left\|f^{n}\right\|_{V^{\prime}}^{p^{\prime}}+\left\|u^{0}\right\|^{p}+\|k\|_{L^{1}(0, T)}^{p}+\|k\|_{L^{p^{\prime}(0, T)}}^{p} \tau \sum_{j=1}^{n-1}\left\|v^{j}\right\|^{p}\right) .
\end{aligned}
$$

Applying a discrete Gronwall-type argument shows that

$$
\left\|v^{n}\right\|^{p} \leq c\left(1+\left\|u^{0}\right\|^{p}+\left\|f^{n}\right\|_{V^{\prime}}^{p^{\prime}}+\tau \sum_{j=1}^{n-1}\left\|f^{j}\right\|_{V^{\prime}}^{p^{\prime}}\right),
$$


and thus

$$
\tau \sum_{j=1}^{n}\left\|v^{j}\right\|^{p} \leq c\left(1+\left\|u^{0}\right\|^{p}+\tau \sum_{j=1}^{n}\left\|f^{j}\right\|_{V^{\prime}}^{p^{\prime}}\right),
$$

which implies the assertion because, similarly as before,

$$
\left\|u^{n}\right\|^{p} \leq c\left(1+\left\|u^{0}\right\|^{p}+\|k\|_{L^{1}(0, T)}^{p}+\|k\|_{L^{p^{\prime}(0, T)}}^{p} \tau \sum_{j=1}^{n}\left\|v^{j}\right\|^{p}\right) .
$$

In view of the growth condition for $B$, we immediately find that, for all $n=1,2, \ldots, N$,

$$
\tau \sum_{j=1}^{n}\left\|B\left(u^{j}, v^{j}\right)\right\|_{V^{\prime}}^{p^{\prime}} \leq c\left(1+\left\|u^{0}\right\|^{p}+\tau \sum_{j=1}^{n}\left\|f^{j}\right\|_{V^{\prime}}^{p^{\prime}}\right) .
$$

This implies, because of (1.2a),

$$
\tau \sum_{j=1}^{n}\left\|A v^{j}\right\|_{V^{\prime}}^{p^{\prime}} \leq c \tau \sum_{j=1}^{n}\left\|f^{j}\right\|_{V^{\prime}}^{p^{\prime}}+c \tau \sum_{j=1}^{n}\left\|B\left(u^{j}, v^{j}\right)\right\|_{V^{\prime}}^{p^{\prime}},
$$

which yields the last a priori estimate.

We shall remark that the second a priori estimate of the proposition above will not be needed in the course of the proof of the main result.

4. Proof of the main result: Convergence of the time discretization. In the sequel, we consider a sequence of time discrete problems (1.2) with step sizes $\tau_{\ell}=T / N_{\ell}$, where $N_{\ell} \in \mathbb{N}$ with $N_{\ell} \rightarrow \infty$ as $\ell \rightarrow \infty$. We recall that $v_{\ell}$ denotes the piecewise constant interpolation of the time discrete solution $\left\{v^{n}\right\}_{n=1}^{N_{\ell}}$ corresponding to the step size $\tau_{\ell}$. Analogously, we denote by $u_{\ell}$ and $f_{\ell}$ the piecewise constant function with $u_{\ell}(t)=u^{n}$ and $f_{\ell}(t)=f^{n}$ if $t \in\left(t_{n-1}, t_{n}\right]\left(n=1,2, \ldots, N_{\ell}\right)$, respectively, where $\left\{f^{n}\right\}_{n=1}^{N_{\ell}}$ is given by (2.3). We set $u_{\ell}(0)=u_{\ell}^{0}$.

Proof of Theorem 2.3. Since the sequence $\left\{u_{\ell}^{0}\right\}$ of starting values for (1.2) is bounded, and since

$$
\left\|f_{\ell}\right\|_{L^{p^{\prime}\left(0, T ; V^{\prime}\right)}}^{p^{\prime}}=\tau_{\ell} \sum_{j=1}^{N_{\ell}}\left\|f^{n}\right\|_{V^{\prime}}^{p^{\prime}} \leq\|f\|_{L^{p^{\prime}\left(0, T ; V^{\prime}\right)}}^{p^{\prime}},
$$


the right-hand side in (3.1a) is uniformly bounded with respect to $\ell$. Moreover, it is easy to prove that

$$
f_{\ell} \longrightarrow f \quad \text { in } L^{p^{\prime}}\left(0, T ; V^{\prime}\right) \text { as } \ell \rightarrow \infty \text {. }
$$

It follows that $\left\{u_{\ell}\right\}$ is bounded in $L^{\infty}(0, T ; V)$ and $\left\{v_{\ell}\right\}$ is bounded in $L^{p}(0, T ; V)$. Hence, there is a subsequence (not relabeled) and elements $u \in L^{\infty}(0, T ; V)$ and $v \in L^{p}(0, T ; V)$ such that

$$
\begin{aligned}
& u_{\ell} \stackrel{*}{\rightarrow} u \quad \text { in } L^{\infty}(0, T ; V) \\
& v_{\ell} \rightarrow v \quad \text { in } L^{p}(0, T ; V) \text { as } \ell \rightarrow \infty,
\end{aligned}
$$

since, in particular, $L^{1}\left(0, T ; V^{\prime}\right)$ is separable and $L^{p}(0, T ; V)$ is reflexive.

For $t \in\left(t_{n-1}, t_{n}\right]\left(n=1,2, \ldots, N_{\ell}\right)$, we observe that

$$
u_{\ell}(t)=u_{\ell}^{0}+\int_{0}^{t_{n}} k\left(t_{n}-s\right) G v_{\ell}(s) d s
$$

and thus,

$$
\begin{aligned}
\left\|u_{\ell}(t)-\left(K G v_{\ell}\right)(t)\right\| \leq & \left\|u_{\ell}^{0}-u_{0}\right\| \\
& +\int_{t}^{t_{n}}\left|k\left(t_{n}-s\right)\right|\left\|G v_{\ell}(s)\right\| d s \\
& +\int_{0}^{t}\left|k\left(t_{n}-s\right)-k(t-s)\right|\left\|G v_{\ell}(s)\right\| d s \\
\leq & \left\|u_{\ell}^{0}-u_{0}\right\|+\left(\int_{t}^{t_{n}}\left|k\left(t_{n}-s\right)\right|^{p^{\prime}} d s\right)^{1 / p^{\prime}} \\
& +\left(\int_{0}^{t}\left|k\left(t_{n}-s\right)-k(t-s)\right|^{p^{\prime}} d s\right)^{1 / p^{\prime}}
\end{aligned}
$$

Because of the growth condition for $G$, the sequence $\left\{G v_{\ell}\right\}$ is bounded in $L^{p}(0, T ; V)$ since $\left\{v_{\ell}\right\}$ does so. The calculation above shows, since $k \in L^{p^{\prime}}(0, T)$, that

$$
\max _{t \in[0, T]}\left\|u_{\ell}(t)-\left(K G v_{\ell}\right)(t)\right\| \longrightarrow 0 \text { as } \ell \rightarrow \infty .
$$


Hence,

$$
u_{\ell}-K G v_{\ell} \longrightarrow 0 \text { in } L^{\infty}(0, T ; V) \text { as } \ell \rightarrow \infty,
$$

and thus $K G v_{\ell}$ converges weakly* in $L^{\infty}(0, T ; V)$ to $u$.

Moreover, since $\left\{G v_{\ell}\right\}$ is bounded in $L^{p}(0, T ; V)$, there is a subsequence (still denoted by $\ell$ ) and $g \in L^{p}(0, T ; V)$ such that

$$
G v_{\ell} \rightarrow g \text { in } L^{p}(0, T ; V) \text { as } \ell \rightarrow \infty .
$$

Since $K: L^{p}(0, T ; V) \rightarrow C([0, T] ; V)$ is affine-linear and bounded (see Proposition 1.1) and thus weakly continuous, we find that

$$
K G v_{\ell} \rightarrow K g \text { in } C([0, T] ; V) \text { as } \ell \rightarrow \infty
$$

as well as

$$
\left(K G v_{\ell}\right)(t) \rightarrow(K g)(t) \text { in } V \text { as } \ell \rightarrow \infty
$$

for all $t \in[0, T]$, which implies with (4.1) that

$$
u_{\ell}(t) \rightarrow(K g)(t) \text { in } V \text { as } \ell \rightarrow \infty
$$

for all $t \in[0, T]$.

Concerning the convergence of $\left\{v_{\ell}\right\}$, we observe the following. Estimate (3.4), together with the boundedness of $\left\{u_{\ell}^{0}\right\}$ in $V$ and (2.3) implies, for all $t \in(0, T]$,

$$
\left\|v_{\ell}(t)\right\| \leq c\left(1+\left\|f_{\ell}(t)\right\|_{V^{\prime}}^{p^{\prime}-1}\right) .
$$

We recall that $f_{\ell}$ converges strongly in $L^{p^{\prime}}\left(0, T ; V^{\prime}\right)$. There is thus a subsequence (still denoted by $\ell$ ) and a majorant $h \in L^{p^{\prime}}(0, T)$ such that

$$
\begin{gathered}
f_{\ell}(t) \longrightarrow f(t) \quad \text { in } V^{\prime} \\
\left\|f_{\ell}(t)\right\|_{V^{\prime}} \leq h(t) \quad \text { for almost all } t \in(0, T) .
\end{gathered}
$$

We thus obtain

$$
\left\|v_{\ell}(t)\right\| \leq c\left(1+h(t)^{p^{\prime}-1}\right) .
$$

For almost all $t \in(0, T)$, we therefore have a subsequence of the subsequence, which we denote by $\ell_{t}^{\prime}$ and which depends on $t$ and an element $v_{t} \in V$ such that

$$
v_{\ell_{t}^{\prime}}(t) \rightarrow v_{t} \quad \text { in } V \text { as } \ell_{t}^{\prime} \rightarrow \infty \text {. }
$$


Later, we shall show that $v_{t}=v(t)$ for almost all $t \in(0, T)$ and that the subsequence is indeed independent of $t$.

The time discrete problem (1.2) can be written as

$$
A v_{\ell}(t)+B\left(u_{\ell}(t), v_{\ell}(t)\right)=f_{\ell}(t) \quad \text { in } V^{\prime}, \quad t \in(0, T)
$$

The strong continuity of $B: V \times V \rightarrow V^{\prime}$ (see Assumption (B)), together with (4.2), (4.3) and (4.5), yields for almost all $t \in(0, T)$ the strong convergence

$$
\begin{gathered}
A v_{\ell_{t}^{\prime}}(t)=f_{\ell_{t}^{\prime}}(t)-B\left(u_{\ell_{t}^{\prime}}(t), v_{\ell_{t}^{\prime}}(t)\right) \rightarrow f(t)-B\left((K g)(t), v_{t}\right):=a_{t} \\
\text { in } V^{\prime} \text { as } \ell_{t}^{\prime} \rightarrow \infty .
\end{gathered}
$$

With Minty's trick (remember that $A: V \rightarrow V^{\prime}$ is monotone and hemicontinuous), we are able to identify the limit $a_{t} \in V^{\prime}$. For arbitrary $z \in V$, we find

$$
\begin{aligned}
\left\langle A v_{\ell_{t}^{\prime}}(t), v_{\ell_{t}^{\prime}}(t)\right\rangle= & \left\langle A v_{\ell_{t}^{\prime}}(t)-A z, v_{\ell_{t}^{\prime}}(t)-z\right\rangle \\
& +\left\langle A v_{\ell_{t}^{\prime}}(t), z\right\rangle+\left\langle A z, v_{\ell_{t}^{\prime}}(t)-z\right\rangle \\
\geq & \left\langle A v_{\ell_{t}^{\prime}}(t), z\right\rangle+\left\langle A z, v_{\ell_{t}^{\prime}}(t)-z\right\rangle .
\end{aligned}
$$

Taking the limit, we obtain

$$
\left\langle a_{t}, v_{t}\right\rangle \geq\left\langle a_{t}, z\right\rangle+\left\langle A z, v_{t}-z\right\rangle .
$$

With $z=v_{t} \pm \theta w$ for arbitrary $w \in V$ and $\theta \in(0,1]$, we thus have

$$
\pm\left\langle A\left(v_{t} \pm \theta w\right), w\right\rangle \geq \pm\left\langle a_{t}, w\right\rangle,
$$

and the hemicontinuity shows, for $\theta \rightarrow 0$, that $a_{t}=A v_{t}$ in $V^{\prime}$.

In view of Assumption (AB), the element $v_{t} \in V$ is the unique solution to

$$
A v_{t}+B\left((K g)(t), v_{t}\right)=f(t) \quad \text { in } V^{\prime}
$$

for given $(K g)(t) \in V$ and $f(t) \in V^{\prime}$. Therefore, by contradiction, the convergence (4.5) not only takes place for a subsequence $\ell_{t}^{\prime}$ depending on $t$ but for the whole sequence for which (4.4) holds. This implies that $\left\{v_{\ell}(t)\right\}$ converges weakly in $V$ towards $v_{t}$ for almost all $t \in(0, T)$. Since we already know that $\left\{v_{\ell}\right\}$ converges weakly in $L^{p}(0, T ; V)$ towards $v$, we find that $v_{t}=v(t)$ for almost all $t \in(0, T)$. 
Let us summarize that $v_{\ell}(t) \rightarrow v(t)$ in $V$ for almost all $t \in(0, T)$ and thus $G v_{\ell}(t) \rightarrow G v(t)$ in $V$ for almost all $t \in(0, T)$ since $G: V \rightarrow V$ is weakly continuous. Moreover, $G v_{\ell} \rightarrow g$ in $L^{p}(0, T ; V)$. This implies $g=G v$, which in our situation can easily be seen as follows. Let $z \in L^{p^{\prime}}\left(0, T ; V^{\prime}\right)$. Then $\left\langle z(t), G v_{\ell}(t)\right\rangle$ converges to $\langle z(t), G v(t)\rangle$ for almost all $t \in(0, T)$. Moreover, we have

$$
\left|\left\langle z(t), G v_{\ell}(t)\right\rangle\right| \leq\|z(t)\|_{V^{\prime}}\left\|G v_{\ell}(t)\right\|_{V} \leq c\|z(t)\|_{V^{\prime}}(1+\|h(t)\|),
$$

where $h \in L^{p^{\prime}}(0, T)$. Lebesgue's theorem on dominated convergence now yields the convergence of $\left\langle z, G v_{\ell}\right\rangle$ to $\langle z, G v\rangle$ in $L^{1}(0, T)$, which means that $G v_{\ell} \rightarrow G v$ in $L^{p}(0, T ; V)$.

This finally shows that $v$ is a solution to (1.1) in the sense of Definition 2.2. Note that $f \in L^{p^{\prime}}\left(0, T ; V^{\prime}\right)$ and, in view of the growth condition (2.1), also $B(K G v, v) \in L^{p^{\prime}}\left(0, T ; V^{\prime}\right)$.

Regarding the regularity statement, we observe the following: If $f \in L^{\infty}\left(0, T ; V^{\prime}\right)$, then (3.4) already implies the boundedness and thus (passing to a subsequence if necessary) the weak*-convergence of $\left\{v_{\ell}\right\}$ in $L^{\infty}(0, T ; V)$ such that $v \in L^{\infty}(0, T ; V)$. Hence, in view of the growth condition (2.1), it follows $B(K G v, v) \in L^{\infty}\left(0, T ; V^{\prime}\right)$. This, finally, implies $A v \in L^{\infty}\left(0, T ; V^{\prime}\right)$.

\section{REFERENCES}

1. C. Bauzet and G. Vallet, On abstract Barenblatt equations, Diff. Equat. Appl. 3 (2011), 487-502.

2. C. Corduneanu, Functional equations with causal operators, Taylor \& Francis, London, 2002.

3. , Abstract Volterra equations: A survey, Math. Comp. Model. 32 (2000), 1503-1528.

4. J. Diestel and J.J. Uhl, Vector measures, American Mathematical Society, Providence, Rhode Island, 1977.

5. K. Diethelm, The analysis of fractional differential equations: An applicationoriented exposition using differential operators of Caputo type, Lect. Notes Math. 2004, Berlin, Springer, 2010.

6. E. Emmrich and G. Vallet, Existence via time discretization for a class of doubly nonlinear operator-differential equations of Barenblatt-type, J. Diff. Equat. 254 (2013), 2499-2514.

7. G. Gripenberg, S.-O. Londen, and O. Staffans, Volterra integral and functional equations, Cambridge Univ. Press, Cambridge, 1990. 
8. R.E. Hilfer, Applications of fractional calculus in physics, World Scientific, Singapore, 2000.

9. A.A. Kilbas, H.M. Srivastava and J.J. Trujillo, Theory and applications of fractional differential equations, Elsevier, Amsterdam, 2006.

10. C. Lubich, Discretized fractional calculus, SIAM J. Math. Anal. 17 (1986), $704-719$.

11. Convolution quadrature revisited, BIT 44 (2004), 503-514.

12. I. Podlubny, Fractional differential equations, Academic Press, San Diego, 1999.

13. J. Prüß, Evolutionary integral equations and applications, Birkhäuser, Basel, 1993.

14. T. Roubíček, Nonlinear partial differential equations with applications, 2nd edition, Birkhäuser, Basel, 2013.

Technische Universität Berlin, Institut für Mathematik, Strasse des 17. Juni 136, 10623 Berlin, Germany

Email address: emmrich@math.tu-berlin.de

Lab. De Mathématiques et Applications de Pau, UMR CNRS 5142, IPRA BP 1155, 64013 Pau Cedex, France

Email address: guy.vallet@univ-pau.fr 